\title{
Optical Flashes and Radio Flares in GRB Afterglows: Numerical Study
}

\author{
Shiho Kobayashi ${ }^{1}$ and Re'em Sari ${ }^{2}$ \\ 1 Department of Earth and Space Science, Osaka University, Toyonaka, Osaka 560, Japan \\ ${ }^{2}$ Theoretical Astrophysics 130-33, California Institute of Technology, Pasadena, CA 91125, USA
}

\begin{abstract}
We numerically study the evolution of an adiabatic relativistic fireball interacting with an ambient uniform medium, both in the initial energy transfer stage and in its late evolution. It is shown that the Blandford-McKee solution adequately describes the evolution of the shocked ejecta quite early on and for as long as the fireball material has relativistic temperatures. In the case where the reverse shock is only mildly relativistic, the shocked ejecta becomes cold almost immediately and the evolution deviates from the Blandford-McKee solution. We derive analytical expressions for the ejecta evolution in its cold regime. This solution gives a good approximation to the numerical results. We estimate the radiation from the fireball ejecta using the numerical hydrodynamic evolution in both cases: cold and hot ejecta. Surprisingly, we find that both evolutions give rather similar light curves, decaying approximately as $t^{-2}$ in the optical and peaking after about one day in the radio, even though the hydrodynamics is different.
\end{abstract}

\section{INTRODUCTION}

A new clue to understand the nature of gamma-ray bursts was found in the event of GRB 990123 by ROTSE which detected a strong optical flash during the "gamma-ray" burst. The optical flash reached to a peak of 9 th magnitude and then decayed with a slope of a power law index $\sim 2[1]$.

Such a strong, prompt optical flash was predicted $[5,7]$. The prompt optical flash observed by ROTSE is compatible with these predictions $[6,8]$. In the energy transfer stage of a fireball evolution, the forward shocked ISM and the reverse shocked fireball cjecta carry comparable amount of internal energy. The typical temperature in the shocked ejecta is considerably lower than that of the shocked ISM, the typical frequency of the emission from the shocked ejecta comes to the optical band with reasonable values of the parameters.

Besides the optical flash, GRB 990123 had another newly observed phenomenon, a radio flare [1]. Sari and Piran [8] have interpreted this flare as the emission from the ejecta particles earlier shocked by the reverse shock. The same particles

CP526, Gamma-Ray Bursts: $5^{\text {th }}$ Huntsville Symposium, edited by R. M. Kippen, et al. (c) 2000 American Institute of Physics 1-56396-947-5/00/ $\$ 17.00$ 
producing the prompt optical flash have been cooled adiabatically and their emission shifts quickly towards lower frequencies while weakening. According to the analytical estimates, scaling the prompt optical emission to the epoch of the radio detection gives the right amount of radio emission.

Although the discussion of the prompt optical flash is quite robust, the estimate of the hydrodynamic evolution of the shocked ejecta is more fragile. In this paper we study numerically the evolution of the ejecta in order to estimate the decay rate of the optical flash as well as the light curve and timing of the radio flare.

\section{THE SHOCKED EJECTA}

Sari and Piran $[7,8]$ used the Blandford-McKee (BM) solution to estimate hydrodynamics of the shocked ejecta. However, it is not clear whether the BM solution is applicable to the shocked ejecta for the following reasons: (i) The BM solution, as a self similar solution, describes the shocked ISM long after the energy transfer stage where the details of the initial conditions are no longer important. (ii) It assumes that the initial ejecta is irrclevant, however the evolution of that ejecta is what we are interested in. (iii) The initial ejecta contains many more particles than those collected by the forward shock. Its density therefore must be higher than that predicted by the BM solution. (iv) Though the BM solution assumes relativistic temperatures, a mildly relativistic reverse shock can not heat ejecta well.

It can be argued that since the shocked ejecta is located not too far behind the forward shock at the end of the energy transfer stage and it has a comparable amount of energy to that of the system, it roughly fits the BM solution. The fact that its rest mass density is much higher than that given by the BM solution should not play an important role as long as the temperature is relativistic. At relativistic temperature, the fluid inertia is due to its thermal energy rather than the rest mass.

The evolution of an adiabatic spherical fireball with an energy $E$, a dimensionless entropy $\eta$ and a radius $R_{0}$ surrounded by a uniform medium with a low density $\rho_{1}$ can be classified into two categories by a dimensionless quantity $\xi \equiv\left(E / \rho_{1} R_{0}^{3}\right)^{1 / 6} / \eta^{4 / 3}$ [2]. If $\xi>1$ the reverse shock is initially Newtonian and becomes mildly relativistic when the reverse shock crosses the ejecta while the reverse shock becomes relativistic during the crossing if $\xi<1$.

Using numerical calculation we verified that the BM solution adequately describes the evolution of the ejecta if the reverse shock is relativistic [3]. In this paper we discuss only the evolution of the cold shell (the case of $\xi>1$ ). We can roughly estimate the evolution of cold ejecta as follows: When the ejecta arrives at radius $R$, the time in the local frame is $R / c \gamma$. The ejecta sound speed is sub-relativistic and can be estimated by $(p / \rho)^{1 / 2}$. The width of the ejecta will be $(p / \rho)^{1 / 2} R / c \gamma$. The ejecta density is therefore: $\rho \propto R^{-3} \gamma(\rho / p)^{1 / 2}$. Using the adiabatic expansion law and assuming a power law $\gamma \propto R^{-g}$, we get $p \propto R^{-8(3+g) / 7}$ and $\rho \propto R^{-6(3+g) / 7}$.

We compare numerical results with this estimate and the BM solution in Figure 1. 
The numerical scaling of the ejecta (thick solid line) is very different from the BM one for a fluid element (dashed line). The power law estimates (thin solid line) are good approximation as long as the bulk motion is relativistic.

\section{THE EJECTA EMISSION}

We estimate in this section the light curve of the optical flash and the radio flare of GRB 990123. According to the internal shock model, the duration of the gamma-ray burst is determined by the thickness of the relativistic flow. We assume $R_{0} / c=40 \mathrm{~s}$. The observations suggest that the typical synchrotron frequency of
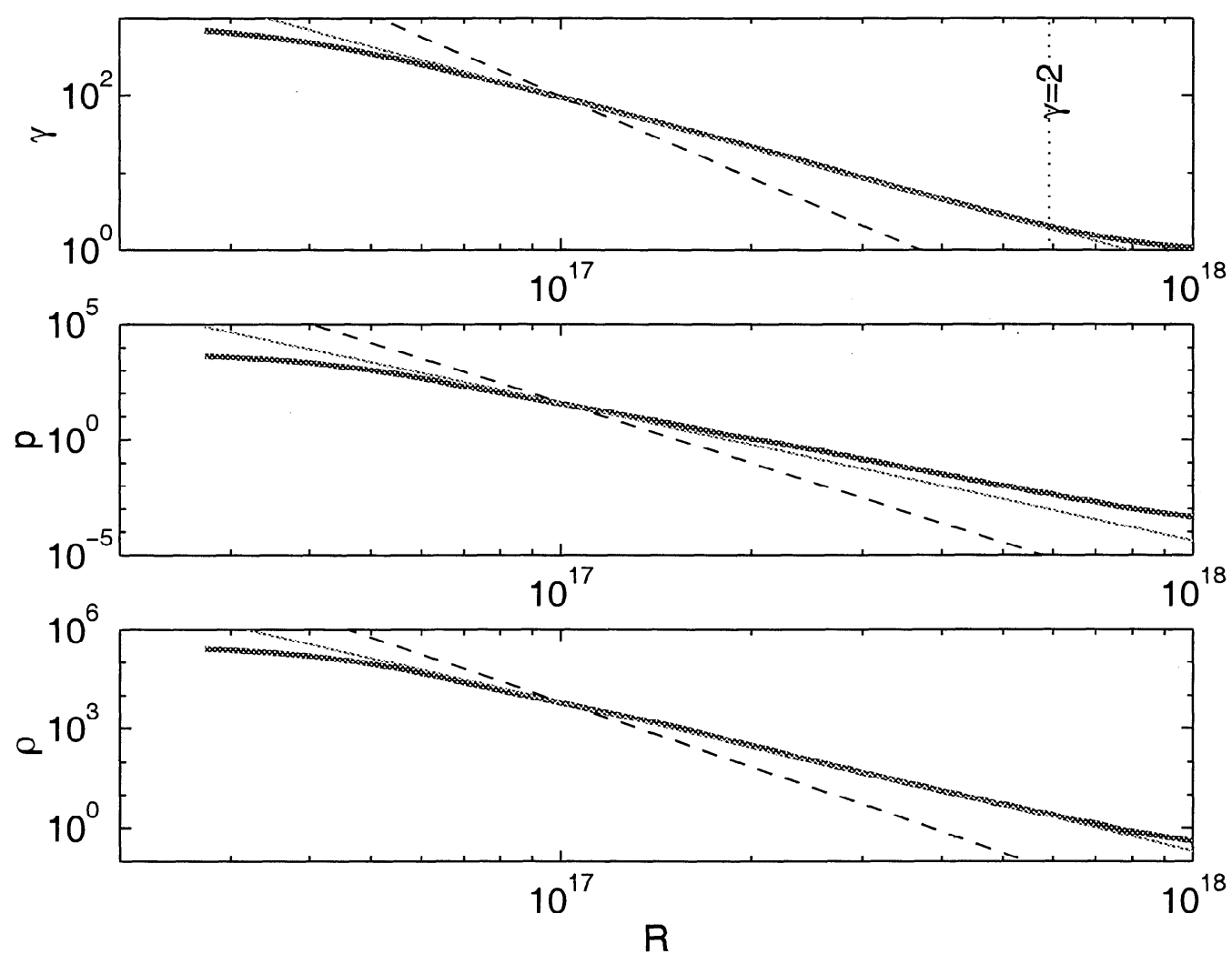

FIGURE 1. R vs Lorentz factor, pressure and density of the ejecta: Newtonian reverse shock case of $\xi=44, E=3 \times 10^{54} \mathrm{erg}, \rho_{1}=10$ proton $/ \mathrm{cm}^{3}, \eta=10^{3}$ and $R_{0}=3 \times 10^{7} \mathrm{~cm}$. The numerical results (thick solid lines) are compared with the Blandford-McKee scalings of a fluid element (dashed lines) and the power law estimates (thin solid lines). The vertical dotted line show the radius where the Lorentz factor of the ejecta is 2 
the reverse shock is below the optical bands quite early on, then the initial Lorentz factor of the ejecta is a few hundred [8]. $\eta=400$ is assumed here. The explosion energy and the ambient density are rather ambiguous. We assume $E=1 \times 10^{54}$ ergs and $\rho_{1}=5$ protons $\mathrm{cm}^{-3}$. The fireball of GRB 990123 is a marginal case of $\xi=0.7$. However, such a marginal case behaves very much like a case of $\xi>>1$ as the ejecta becomes cold at the early stage.

In order to estimate the light curve from the thermodynamic quantities of the fireball ejecta, we assume that the energy of the magnetic field and the internal energy of electrons remain constant fractions of the internal energy of the fluid. If the shocked electrons in the shocked ejecta are accelerated into the power law distribution of the electron random Lorentz factor with index $\hat{p}$, the spectral flux at a given frequency above the typical frequency $\nu_{m}$ is $F_{\nu} \sim F_{\nu_{m}}\left(\nu / \nu_{m}\right)^{-(\hat{p}-1) / 2}$.

Using the numerical evolution of $\gamma, p$ and $\rho$, the optical light curve is plotted in Figure 2. The ROTSE observations are also plotted (stars), the triangles represent upper limits. The steepness of the light curve is about -2 at late time with a steeper slope at early times. The numerical light curve normalized at the radio flare reasonably fits the observation.

Emission from the reverse shock can also explain the radio flare, the radio detection one day after the burst. The shocked ejecta initially radiates in the optical band. As the ejecta expands, the temperature of the ejecta becomes lower. The emission frequency and the flux drop quickly; eventually the emission comes into the radio band. The flux at $8.5 \mathrm{GHz}$ is plotted in Figure 2 in which we consider the upper limit by the self absorption (dashed line) [3]. The numerical light curves fit to both the optical flash and the radio flare.

\section{DISCUSSION}

We have seen that the Blandford-McKee solution is not applicable to the shocked ejecta if the reverse shock is Newtonian or mildly relativistic and the temperature of the shocked ejecta is not relativistic. The hydrodynamics of the cold shocked ejecta are very different from that of the hot shocked ejecta which is well described by the Blandford-McKee solution. The numerical scaling relations for a cold one were well approximated by the spreading assumption and the adiabatic expansion law, while the Blandford-McKee solution satisfies the relativistic version of it. We have estimated the radiation from the fireball ejecta. Surprisingly, we find that the evolution of both the cold and hot ejecta give rather similar light curves of optical and radio even though the hydrodynamics is very different.

\section{REFERENCES}

1. Akerlof, C.W., et al., Nature 398, 400 (1999).

2. Kobayashi, S., Piran,T. and Sari, R., ApJ 513, 669 (1999).

3. Kobayashi S. and Sari R., $A p J$ submitted (1999) astro-ph/9910241. 
4. Kulkarni, S.R., et al., Nalure 398, 389(1999).

5. Mészaros, P. and Rees,M.J., ApJ 476, 231 (1997).

6. Mészaros, P. and Rees,M.J., MNRAS submitted (1997) astro-ph/9902367.

7. Sari, R. and Piran,T., ApJ 520, 641(1999).

8. Sari, R. and Piran,T., ApJ 517, L109(1999).
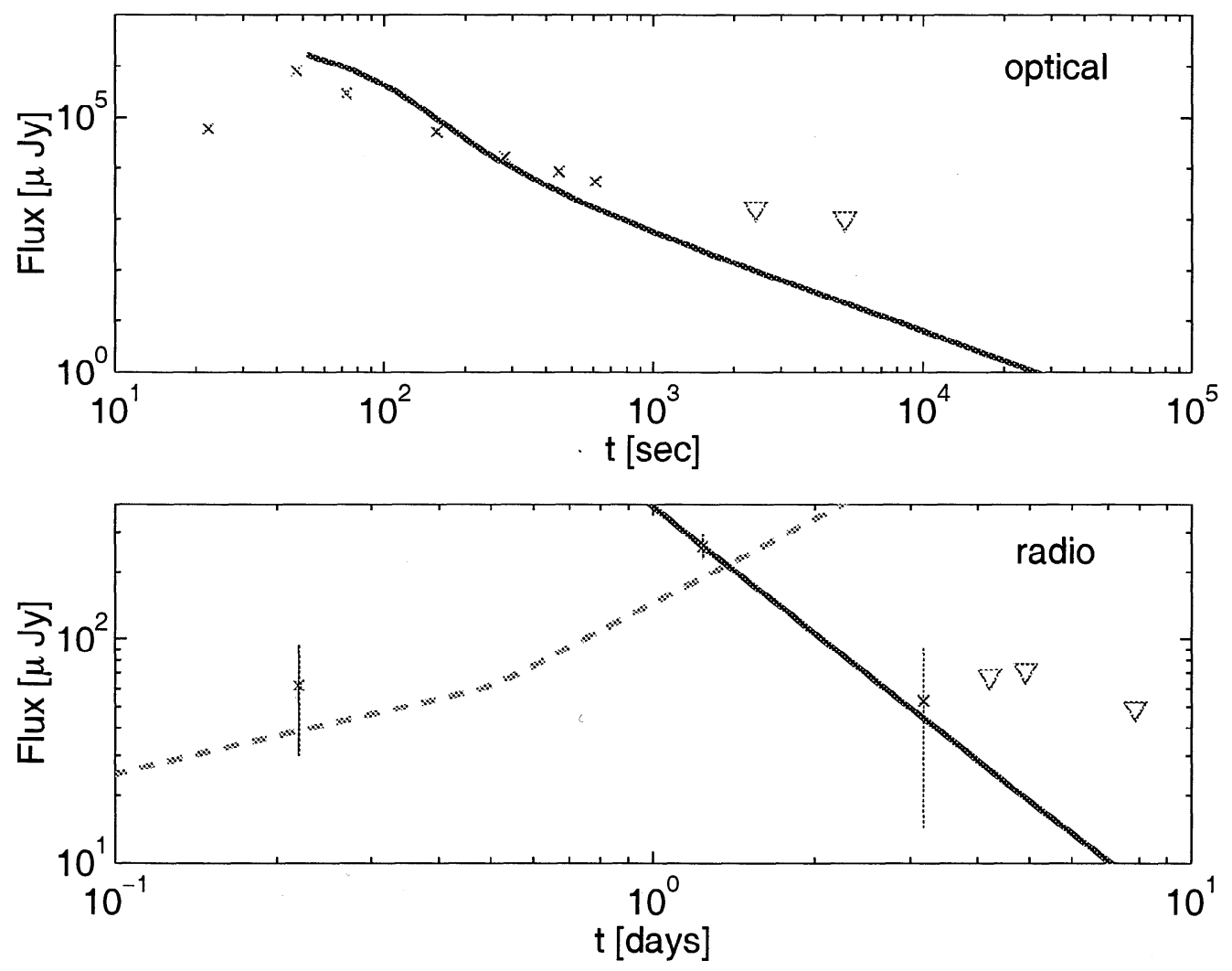

FIGURE 2. Light curves of optical flash and radio flare. The solid lines depicts the numerical light curves. The stars are the observations and the triangles are upper limits. The dashed line is self absorption upper limit. 